\title{
Pacific
}

Journal of

Mathematics

\section{ON THE EXISTENCE OF EXTREMAL METRICS}

XINGWANG XU 


\title{
ON THE EXISTENCE OF EXTREMAL METRICS
}

\author{
XINGWANG XU
}

We study the well known variational problem proposed by Calabi: Minimize the functional $\int_{M} s_{g}^{2} d v_{g}$ among all metrics in a given Kahler class. We are able to establish the existence of the extremal when the closed Riemann surface has genus different from zero. We have also given a different proof of the result originally proved by Calabi that: On a closed Riemann surface, the extremal metric has constant scalar curvature on a closed Riemann surface, the extremal metric has constant scalar curvature, which originally is proved by Calabi.

\section{Introduction.}

In the early 80 's, E. Calabi $[\mathbf{C 1}, \mathbf{C 2}]$ proposed the following variational problem. Let $M$ be a compact, connected, complex $n$-dimensional manifold without boundary and assume that $M$ admits a Kähler metric $g$ locally expressible in the form $d s^{2}=2 g_{\alpha \bar{\beta}} d z^{\alpha} d z^{\bar{\beta}}$. Let us fix the deRham cohomology class $\Omega$ of the real valued, closed exterior $(1,1)$ form $\omega=\sqrt{-1} g_{\alpha \bar{\beta}} d z^{\alpha} \Lambda d z^{\bar{\beta}}$ associated to the metric $g$, and denote by $\mathcal{C}_{\Omega}$ the function space of all differentiable Kähler metrics $g$ with the Kähler form $\omega \in \Omega$. On this function space, Calabi introduces the (non negative) real valued functional $\Phi$ which assigns to each $g$ the integral

$$
\Phi(g)=\int_{M} s_{g}^{2} d v_{g}
$$

where $d v_{g}=(\sqrt{-1})^{n} \operatorname{det}\left(g_{\alpha \bar{\beta}}\right) \Lambda_{\alpha=1}^{n}\left(d z^{\alpha} \Lambda d z^{\bar{\alpha}}\right)$ denotes the volume element in $M$ associated with the e Kähler metric $g$, and

$$
s_{g}=-g^{\alpha \bar{\beta}} \frac{\partial^{2}}{\partial z^{\alpha} \partial z^{\bar{\beta}}} \log \operatorname{det}\left(g_{\lambda \bar{\mu}}\right)
$$

the scalar curvature.

The variational problem proposed by Calabi is that of minimizing the functional $\Phi(g)$ over all $g \in \mathcal{C}_{\Omega}$. The motivation for considering this is the fact that, as $g$ varies in $\mathcal{C}_{\Omega}$, both the volume

$$
V=V_{g}=\int_{M} d v_{g}
$$


and the total scalar curvature

$$
S_{g}=\int_{M} s_{g} d v_{g}
$$

remain constants. Thus by the virtue of the Schwartz inequality, the functional $\Phi(g)$ has a nonnegative lower bound $S_{g}^{2} V$, we wish that the latter can be achieved if and only if there exists a $g \in \mathcal{C}_{\Omega}$ with constant scalar curvature.

As M. Levine $[\mathbf{L}]$ points out, if we call the critical metric of $\Phi$ the extremal metric, the extremal metric does not necessarily have constant scalar curvature if the dimension $n>1$. For $n=1$, E. Calabi is able to show that the extremal metric always has constant scalar curvature if the extremal metric exists (see [C1] and also $\S 5$ of this paper).

The problem of finding extremal metrics is quite nature but quite difficult. There are severval results about the non-existence $([\mathbf{C 1}],[\mathbf{C 2}],[\mathbf{L}],[\mathbf{B B}])$. However, in the past decade, there has almost been no progress on the existence of extremal metrics.

The propose of this paper is to show:

Main Theorem.If $n=1$ with $\chi(M) \leq 0$, then the extremal metric exists.

Remember that this is not so surprising at all since there are several methods to reach this conclusion: Poincaré's classical uniformization theorem $[\mathbf{P}]$; M. Berger's minimization method $[\mathbf{A}],[\mathbf{B}]$; R. Hamilton's Ricci flow $[\mathbf{C H}],[\mathbf{H 1}],[\mathbf{H 2}]$; B. Osgood, R. Phillips and P. Sarnack's minimizing the $\log$ determinent of the Laplace operator [OPS].

What it is new in this paper is that, we exactly follow the Calabi's original idea, by using the direct method, to show that the minimizer of the Calabi functional can be achieved. Since the Kähler class and the conformal class for $n=1$ are equivalent, our setting is in the conformal class. The main difficulty is to get $H_{2}^{2}$ norm bound of the conformal factors in terms of the volume bound and the bound on the Calabi functional.

The organization of this paper is as follows: after some preliminaries $(\S 2)$, we will give the proof of our main theorem for the case $\chi(M)<0(\S 3)$. $\S 4$ will simply indicate the case $\chi(M)=0$. Since our setting is in the conformal class, we will show, in this setting, that the extremal metrics have constant scalar curvatures ( $(5)$. Clearly this is an alternative proof of one of Calabi's theorems [C1].

The author would like to thank Professor Paul Yang for letting him know this very interesting problem and Professor William Abikoff for an encouragement on him to write out this proof. 


\section{Preliminaries.}

Consider a compact connected complex $n$-dimensional manifold $M$ without boundary. Assume that $M$ admits a Kähler metric $g$ locally expressible in the form

$$
d s^{2}=2 g_{\alpha \bar{\beta}} d z^{\alpha} d z^{\bar{\beta}}
$$

where and thereafter the Einstein convention is used. It is well known that there is a real valued, closed exterior $(1,1)$ form $\omega=\sqrt{-1} g_{\alpha \bar{\beta}} d z^{\alpha} d z^{\bar{\beta}}$ associated to the metric $g$. This $(1,1)$ form usually is called Kähler form. By the deRham theory, $\omega$ determines a deRham cohomology class $\Omega$. Now let us consider the change of the Kähler metric,

$$
g_{\lambda \bar{\mu}}^{\prime}=g_{\lambda \bar{\mu}}+\partial_{\lambda \bar{\mu}} \varphi
$$

where $\varphi \in C^{\infty}$ is such that $g^{\prime}$ is positive definite. Obviously, $g^{\prime}$ is a Kähler metric. Also it is clear that the Kähler form $\omega^{\prime}$ associated to the metric $g^{\prime}$ determines the same deRham cohomology class as $\omega$ does. Note that under this change of the metric, new metric $g^{\prime}$ is also a Riemannian metric since it is Kähler. We will denote all such functions $\varphi$ by $\mathcal{C}_{\Omega}$.

For a given Kähler metric $g$, we have the volume element defined in the local coordinate by $d v_{g}=(\sqrt{-1})^{n} \operatorname{det}\left(g_{\alpha \bar{\beta}}\right) \Lambda_{\alpha=1}^{n}\left(d z^{\alpha} \Lambda d z^{\bar{\alpha}}\right)$. And the scalar curvature associated to the metric $g$ is defined by

$$
s_{g}=-g^{\alpha \bar{\beta}} \frac{\partial^{2}}{\partial z^{\alpha} \partial z^{\bar{\beta}}}\left(\log \operatorname{det}\left(g_{\lambda \bar{\mu}}\right)\right) .
$$

For a $\varphi \in \mathcal{C}_{\Omega}$, the Calabi functional can be written as

$$
\Phi(\varphi)=\int_{M} s_{g^{\prime}}^{2} d v_{g^{\prime}}
$$

where $g^{\prime}$ and $\varphi$ are related by (2.1).

From now on, we only consider $M$ a Riemann surface without boundary, that is, a compact complex 1-dimensional manifold without boundary. It is known that on a complex 1-dimensional manifold, any Riemannian metric is conformally flat [Be, Theorem 1.169]. Thus any two Riemannian metrics on $M$ are conformally equivalent. If metrics $g$ and $g^{\prime}$ are related by (2.1), there exists a $C^{\infty}$ function $u$ such that $g^{\prime}=e^{2 u} g$. In this form, we know that $d v_{g^{\prime}}=e^{2 u} d v_{g}$ and the scalar curvatures satisfy the relation

$$
\Delta u+k_{g^{\prime}} e^{2 u}=k_{g}
$$

where $k_{g^{\prime}}$ and $k_{g}$ are the Gaussian curvatures of the metrics $g^{\prime}$ and $g$ respectively, and $\Delta$ is the Laplace operator associated to the metric $g$. The relation 
between the Gaussian curvature and the scalar curvature is that $s_{g}=2 k_{g}$. Thus, up to a constant multiple, the Calabi functional $\Phi(\varphi)$ is equivalent to

$$
J(u)=\int_{M}\left(k_{g}-\Delta u\right)^{2} e^{-2 u} d v_{g}
$$

with the constrain

$$
\int_{M} e^{2 u} d v_{g}=\int_{M} d v_{g}
$$

Our main propose of this section is going to show that the functional $J$ has the following properties.

\section{Proposition 2.1.}

(a) $J$ is continuously differentiable on $H_{2}^{2}$ where $H_{2}^{2}$ denotes the Hilbert space as usual.

(b) The first variation of $J$ at a point $u$ in a direction $\varphi$ is given by

$$
J^{\prime}(u)(\varphi)=-2 \int_{M}\left[e^{2 u}\left(k_{g}-\Delta u\right)^{2}+\Delta\left(e^{2 u}\left(k_{g}-\Delta u\right)\right)\right] \varphi d v_{g} .
$$

(c) The Euler equation associated to $J$ under the constrain (1.6) is given by

$$
\Delta\left[e^{-2 u}\left(k_{g}-\Delta u\right)\right]+e^{-2 u}\left(k_{g}-\Delta u\right)^{2}=\lambda^{2} e^{2 u}
$$

for some constant $\lambda \geq 0$.

Proof. (c) can easily follow from (b), Lagrange multiplier and the fact that if we set $G(u)=\int_{M} e^{2 u} d v_{g}, G^{\prime}(u)(\varphi)=\int_{M} e^{2 u} \varphi d v_{g}$. (b) will follow from the proof of (a). Before we are going to prove the part (a), let us recall two simple facts.

Fact 1. If the real dimension of a manifold is two, there exists a constant $C>0$ such that

$$
\max |u| \leq C\|u\|_{H_{2}^{2}}
$$

for all $u \in H_{2}^{2}$.

It can be proved as follows. Suppose $G$ is a Green function for $\Delta$. Then it is known that $\int_{M} G^{2} d v_{g}$ is finite and for any $u \in H_{2}^{2}, u(p)=\int_{M} u d v_{g}$ $\int_{M} G \Delta u d v_{g}$. Thus the Hölder inequality implies that (2.9) holds with $C=$ $\left[\left(\int_{M} d v_{g}\right)^{1 / 2}+\left(\int_{M} G^{2} d v_{g}\right)^{1 / 2}\right]$.

Fact 2. We can choose $\varepsilon_{0}>0$ such that if $0<t<\varepsilon_{0}$, then $\left|e^{-2 t}-1+2 t\right| \leq 4 t^{2}$ and $\left|e^{-2 t}-1\right| \leq 100 t$ where numbers 4 and 100 are not so important. 
In order to simplify the notation, from now on, we will denote the norm $\|u\|_{H_{2}^{2}}$ by $\|u\|$.

Now we are in position to give the proof of the part (a).

Let us now assume that $u \in H_{2}^{2}$ such that $J(u)<\infty$. Let $\varphi$ be any function in $H_{2}^{2}$ such that $\max |\varphi|<\epsilon_{0}$. Then we have

$$
\begin{aligned}
\|\varphi\|^{-1}\left|J(u+\varphi)-J(u)+2 \int_{M}\left[e^{-2 u}\left(k_{g}-\Delta u\right)^{2}+\Delta\left(e^{-2 u}\left(k_{g}-\Delta u\right)\right)\right] \varphi d v_{g}\right| \\
=\|\varphi\|^{-1} \mid \int_{M}\left(k_{g}-\Delta u\right)^{2} e^{-2 u}\left[e^{-2 \varphi}+2 \varphi-1\right] d v_{g} \\
\quad+\int_{M}(\Delta \varphi)^{2} e^{-2(u+\varphi)} d v_{g}-2 \int_{M} \Delta \varphi\left[e^{-2 u}\left(k_{g}-\Delta u\right)\right]\left(e^{-2 \varphi}-1\right) d v_{g} \mid \\
\leq\|\varphi\|^{-1}\left[4 C^{2}\|\varphi\|^{2} J(u)+C_{1} \exp [2 C(\|u\|+\|\varphi\|)] \cdot\|\varphi\|^{2}\right. \\
\left.\quad+200 C_{2} \exp [C\|u\|] J(u)^{\frac{1}{2}} \cdot\|\varphi\|^{2}\right] \\
=\left[4 C^{2} J(u)+C_{1} \exp [2 C(\|u\|+\|\varphi\|)]+200 C_{2} \exp [C\|u\|] J(u)^{\frac{1}{2}}\right] \cdot\|\varphi\|,
\end{aligned}
$$

where $C$ is given in (2.9) and $C_{1}$ and $C_{2}$ are constants. This proves (b) and the half of (a). For the rest of the part (a), we can argue as follows: for a fixed function $u \in H_{2}^{2}$, if $v \in H_{2}^{2}$ is such that $\max |u-v|<\varepsilon_{0}$, then we have

$$
\begin{aligned}
& \left|J^{\prime}(u)(w)-J^{\prime}(v)(w)\right| \\
& =2 \mid \int_{M}\left[e^{-2 u}\left(k_{g}-\Delta u\right)^{2}+\Delta\left(e^{-2 u}\left(k_{g}-\Delta u\right)\right)\right] w d v_{g} \\
& -\int_{M}\left[e^{-2 v}\left(k_{g}-\Delta v\right)^{2}+\Delta\left(e^{-2 v}\left(k_{g}-\Delta v\right)\right)\right] w d v_{g} \\
& =2 \mid \int_{M}\left(e^{-2 u}-e^{-2 v}\right)\left(k_{g}-\Delta u\right)^{2} w d v_{g} \\
& +\int_{M} e^{-2 v}\left[\left(k_{g}-\Delta u\right)^{2}-\left(k_{g}-\Delta v\right)^{2}\right] w d v_{g} \\
& +\int_{M}\left(e^{-2 u}-e^{-2 v}\right) k_{g} \Delta w d v_{g}-\int_{M}\left(e^{-2 u} \Delta u-e^{-2 v} \Delta v\right) \Delta w d v_{g} \\
& \leq 200 \max |u-v| J(u) \max |w|+2 e^{2 \max |u|} \int_{M}|\Delta(u-v)||\Delta w| d v_{g} \\
& +2 e^{2 \max |v|} \max |w| \int_{M}|\Delta(u-v)|\left|2 k_{g}-\Delta(u+v)\right| d v_{g} \\
& +400 e^{\max |v|} \int_{M}|u-v||\Delta w| d v_{g} \max \left|k_{g}\right| \\
& +2 e^{2 \max |u|} \max |u-v| \cdot\|v\| \cdot\|w\| \\
& \leq C\|u-v\| \cdot\|w\|
\end{aligned}
$$


where $C>0$ depends on $\|u\|,\|v\|, J(u)$ and $\max \left|k_{g}\right|$. This proves the result. have

Also we note that if we define $H=\left\{u \in H_{2}^{2} \mid \int_{M} e^{2 u} d v_{g}=\int_{M} d v_{g}\right\}$, we

Proposition 2.2. $H$ is a weakly closed subset in $H_{2}^{2}$.

Proof. Weakly convergent subsequences in $H_{2}^{2}$ is strongly convergent sequences in $H_{1}^{2}$. $\quad \int_{M} e^{2 u} d v_{g}$ is a weakly continuous functional on $H_{1}^{2}$ by Moser's inequality. Thus it is a weakly continuous functional on $H_{2}^{2}$. We are done.

\section{Proof of Main Theorem for $\chi(M)<0$.}

The proof of the main theorem in this case will consist of several lemmas.

Lemma 3.1. Let $(M, g)$ be a closed Riemann surface with $\chi(M)<0$. If the Gaussian curvature $k_{g}$ is positive somewhere, there exists a function $u \in H_{2}^{2}$ so that the metric $\tilde{g}=e^{2 u} g$ has nonpositive Gaussian curvature $k_{\tilde{g}}$ and $\int_{M} k_{\tilde{g}}^{2} d v_{\tilde{g}} \leq \int_{M} k_{g}^{2} d v_{g}$.

Proof. Choose

$$
s= \begin{cases}-k_{g} & k_{g} \geq 0 \\ k_{g} & k_{g} \leq 0 .\end{cases}
$$

Then it is clear that $s \leq 0$. Let us consider

$$
\Delta u+s e^{2 u}=k_{g} .
$$

Clearly, if $\varphi=0$, then

$$
\Delta \varphi+s e^{2 \varphi}-k_{g}= \begin{cases}-2 k_{g} & k_{g} \geq 0 \\ 0 & k_{g} \leq 0\end{cases}
$$

which is always nonpositive. Let $\phi$ be a solution of $\Delta \phi=k_{g}-\frac{\int_{M} k_{g} d v_{g}}{\int_{M} d v_{g}}$. By standard elliptic theory, up to add a constant to $\phi, \phi$ is unique. We fix a solution by requiring $\int_{M} \phi d v_{g}=0$. Choose $N>0$ large enough so that $s e^{2 \phi-N}-\frac{\int_{M} k_{g} d v_{g}}{\int_{M} d v_{g}}>0$ and $\phi-N / 2<0$. It is clear that such an $N$ exists. Then set $v=\phi-N / 2$, we can get

$$
\Delta v+s e^{2 v}-k_{g}=s e^{2 \phi-N}-\frac{\int_{M} k_{g} d v_{g}}{\int_{M} d v_{g}}>0
$$


and $v<0$. Therefore $\phi=0$ is a supper solution and $v$ is a sub-solution of the equation (3.1). By standard elliptic theory, equation (3.1) has a $H_{2}^{2}$ solution $w$ since $s$ and $k_{g}$ are in $L^{2}(M)$, [S, Theorem 2.4; Chapter 1].

Now choose a constant $\alpha$ such that $\int_{M} e^{2(w+\alpha)} d v_{g}=\int_{M} d v_{g}$, and set $\tilde{k}=$ $s e^{-2 \alpha} \leq 0$. Since $w \leq 0$ and $-2 \alpha=\log \frac{\int_{M} e^{2 w} d v_{g}}{\int_{M} d v_{g}}$, by the convexity of the exponential function, $\alpha>0$. Also $\int_{M}(\tilde{k})^{2} e^{2(w+\alpha)} d v_{g}=\int_{M} s^{2} e^{-4 \alpha} e^{2(w+\alpha)} d v_{g}=$ $\int_{M} s^{2} e^{2(w-\alpha)} d v_{g} \leq \int_{M} s^{2} d v_{g}=\int_{M} k_{g}^{2} d v_{g}$. Thus if we set $u=w+\alpha$, then $\tilde{g}=e^{2 u} g$ will satisfy the requirement. This completes the proof of Lemma 3.1 .

Lemma 3.2. If $u \in H_{2}^{2}, J(u) \leq C_{0}, k_{g}-\Delta u \leq 0$ and $\int_{M} e^{2 u} d v_{g}=\int_{M} d v_{g}$, there exist constants $C_{1}, C_{2}$ depending only $C_{0}$ and $k_{g}$ and $\int_{M} d v_{g}$ such that the following inequality holds: $C_{1}<u<C_{2}$.

Proof. Let $\varphi$ be a solution of the equation $\Delta \varphi=k_{g}-\frac{\int_{M} k_{g} d v_{g}}{\int_{M} d v_{g}}$ with $\int_{M} \varphi d v_{g}=$ 0 . Since $k_{g}$ is continuous and $\varphi$ is in $C^{2}, \varphi$ is in $H_{2}^{2}$. There exist constants $\alpha$ and $\beta$ such that $\alpha<\varphi<\beta$ where $\alpha$ and $\beta$ only depend on $k_{g}$.

Now from $J(u) \leq C_{0}$, we have

$$
\begin{aligned}
C_{0} \geq J(u)= & \int_{M} e^{-2 u}\left(k_{g}-\Delta u\right)^{2} d v_{g} \\
\geq & \int_{M} e^{2(\varphi-\beta)}\left(k_{g}-\Delta u\right)^{2} e^{-2 u} d v_{g} \\
= & e^{-2 \beta} \int_{M} e^{-2(u-\varphi)}\left(k_{g}-\Delta u\right)^{2} d v_{g} \\
= & e^{-2 \beta} \int_{M} e^{-2(u-\varphi)}\left(k_{g}-\frac{\int_{M} k_{g} d v_{g}}{\int_{M} d v_{g}}+\frac{\int_{M} k_{g} d v_{g}}{\int_{M} d v_{g}}-\Delta u\right)^{2} \\
= & e^{-2 \beta} \int_{M} e^{-2(u-\varphi)}\left(\frac{\int_{M} k_{g} d v_{g}}{\int_{M} d v_{g}}-\Delta(u-\varphi)\right)^{2} d v_{g} \\
= & e^{-2 \beta}\left[\left(\frac{\int_{M} k_{g} d v_{g}}{\int_{M} d v_{g}}\right)^{2} \int_{M} e^{-2(u-\varphi)} d v_{g}\right. \\
& -2 \frac{\int_{M} k_{g} d v_{g}}{\int_{M} d v_{g}} \int_{M} e^{-2(u-\varphi)} \Delta(u-\varphi) d v_{g} \\
& \left.+\int_{M} e^{-2(u-\varphi)}(\Delta(u-\varphi))^{2} d v_{g}\right] \\
= & e^{-2 \beta}\left[\left(\frac{\int_{M} k_{g} d v_{g}}{\int_{M} d v_{g}}\right)^{2} \int_{M} e^{-2(u-\varphi)}\right.
\end{aligned}
$$




$$
\begin{aligned}
& -4 \frac{\int_{M} k_{g} d v_{g}}{\int_{M} d v_{g}} \int_{M} e^{-2(u-\varphi)}|\nabla(u-\varphi)|^{2} d v_{g} \\
& \left.+\int_{M} e^{-2(u-\varphi)}(\Delta(u-\varphi))^{2} d v_{g}\right]
\end{aligned}
$$

The above inequality and the fact that $\int_{M} k_{g} d v_{g}=\chi(M) \int_{M} d v_{g}<0$ imply that

$$
\begin{aligned}
\int_{M} e^{-2(u-\varphi)} d v_{g} & \leq \frac{\left[C_{0} e^{2 \beta}\left(\int_{M} d v_{g}\right)^{2}\right]}{\left(\int_{M} k_{g} d v_{g}\right)^{2}} \\
\int_{M} e^{-2(u-\varphi)}|\nabla(u-\varphi)|^{2} d v_{g} & \leq \frac{\left[C_{0} e^{2 \beta} \int_{M} d v_{g}\right]}{\left(-4 \int_{M} k_{g} d v_{g}\right)} \\
\int_{M} e^{-2(u-\varphi)}|\Delta(u-\varphi)|^{2} & \leq C_{0} e^{2 \beta}
\end{aligned}
$$

Simply (3.2) gives us

$$
e^{2 \alpha} \int_{M} e^{-2 u} d v_{g} \leq \int_{M} e^{-2 u+2 \varphi} d v_{g} \leq \frac{\left[C_{0} e^{2 \beta}\left(\int_{M} d v_{g}\right)^{2}\right]}{\left(\int_{M} k_{g} d v_{g}\right)^{2}}
$$

or equivalently,

$$
\int_{M} e^{-2 u} d v_{g} \leq \frac{C_{0} e^{2(\beta-\alpha)}\left(\int_{M} d v_{g}\right)^{2}}{\left(\int_{M} k_{g} d v_{g}\right)^{2}}:=C_{3} .
$$

By using the convexity of the exponential function, we can get that $-\int_{M} 2 u d v_{g} \leq \log C_{3}$ from (3.5). Since $\int_{M} e^{2 u} d v_{g}=\int_{M} d v_{g}, 2 \int_{M} u d v_{g} \leq$ $\log \int_{M} d v_{g}:=C_{4}$.

From now on we will assume that the volume $\int_{M} d v_{g}=1$.

Thus $C_{4}=0$. Anyway, we get $\left|\int_{M} u d v_{g}\right| \leq \frac{\log C_{3}}{2}$. Now

$$
u(p)-\int_{M} u d v_{g}=-\int_{M} G \Delta u d v_{g}
$$

where $G$ is Green's function associated to Laplace operator $\Delta$. We can choose $G$ such that it is positive everywhere [A, Theorem 4.14]. These give us the estimate on $u$ as follows

$$
\begin{aligned}
u(p)-\int_{M} u d v_{g} & =\int_{M} G\left(k_{g}-\Delta u\right) d v_{g}-\int_{M} k_{g} G d v_{g} \\
& \leq\left(\int_{M}\left(k_{g}\right)^{2} d v_{g}\right)^{1 / 2}\left(\int_{M} G^{2} d v_{g}\right)^{1 / 2} \\
& :=C_{5} .
\end{aligned}
$$


This implies that

$u(p) \leq\left(\int_{M} u d v_{g}\right)+\left(\int_{M}\left(k_{g}\right)^{2} d v_{g}\right)^{1 / 2}\left(\int_{M} G^{2} d v_{g}\right)^{1 / 2} \leq \frac{\log C_{3}}{2}+C_{5}:=C_{2}$.

As $p$ is arbitrary on $M, u \leq C_{2}$.

Apply (3.6) with $u$ replaced by $e^{-u}$ to get

$$
\begin{aligned}
e^{-u}-\int_{M} e^{-u} d v_{g} & =-\int_{M} G \Delta e^{-u} d v_{g} \\
& =\int_{M} e^{-u} G\left(\Delta u-|\nabla u|^{2}\right) d v_{g} \\
& \leq \int_{M} e^{-u} G \Delta u d v_{g} \\
& \leq\left(\int_{M} e^{-2 u}(\Delta u)^{2} d v_{g}\right)^{1 / 2}\left(\int_{M} G^{2} d v_{g}\right)^{1 / 2}
\end{aligned}
$$

where we have used the fact that $\Delta\left(e^{-u}\right)=e^{-u}\left(|\nabla u|^{2}-\Delta u\right)$. But from (3.3), we obtain

$$
\begin{aligned}
C_{0} e^{2 \beta} \geq & \int_{M} e^{-2(u-\varphi)}(\Delta(u-\varphi))^{2} d v_{g} \\
= & \int_{M} e^{-2(u-\varphi)}\left[(\Delta u)^{2}-2 \Delta u \Delta \varphi+(\Delta \varphi)^{2}\right] d v_{g} \\
\geq & \int_{M} e^{-(u-\varphi)}(\Delta u)^{2} d v_{g}-1 / 2 \int_{M}(\Delta u)^{2} e^{-2(u \varphi)} d v_{g} \\
& -2 \int_{M}(\Delta \varphi)^{2} e^{-2(u-\varphi)} d v_{g}+\int_{M}(\Delta \varphi)^{2} e^{-2(u-\varphi)} d v_{g} \\
= & 1 / 2 \int_{M} e^{-2(u-\varphi)}(\Delta u)^{2} d v_{g}-\int_{M}(\Delta \varphi)^{2} e^{-2(u-\varphi)} d v_{g}
\end{aligned}
$$

This simply implies that

$$
\begin{aligned}
& \frac{1}{2} \int_{M} e^{-2 u}(\Delta u)^{2} d v_{g} e^{2 \alpha} \\
& \leq \frac{1}{2} \int_{M} e^{-2(u-\varphi)}(\Delta u)^{2} d v_{g} \\
& \leq \int_{M} e^{-2(u-\varphi)}(\Delta(u-\varphi))^{2} d v_{g}+\int_{M} e^{-2(u-\varphi)}(\Delta \varphi)^{2} d v_{g} \\
& \leq C_{0} e^{2 \beta}+\int_{M}\left(\int_{M} k_{g} d v_{g}-k_{g}\right)^{2} \cdot e^{-2 u} d v_{g} \cdot e^{2 \beta} \\
& \leq\left[C_{0}+2\left(\int_{M} k_{g} d v_{g}\right)^{2} \int_{M} e^{-2 u} d v_{g}+2 \int_{M} k_{g}^{2} e^{-2 u} d v_{g}\right] e^{2 \beta}
\end{aligned}
$$




$$
\begin{aligned}
& \leq\left\{C_{0}+2\left[\left(\int_{M} k_{g} d v_{g}\right)^{2}+\left(\max \left|k_{g}\right|\right)^{2}\right] C_{3}\right\} e^{2 \beta} \\
& :=C_{6}
\end{aligned}
$$

by using (3.5) and (3.7). Thus this implies that

$$
\begin{aligned}
e^{-u(p)} & \leq C_{3}^{1 / 2}+\left(\int_{M} G^{2}\right)^{1 / 2}\left(2 C_{6}\right)^{1 / 2} e^{-\alpha} \\
& :=e^{-C_{1}} .
\end{aligned}
$$

Hence, by taking log on both sides of (3.9), we have $u(p) \geq C_{1}$. As before, $p$ is a general point on $M$, we have what we need to show.

Lemma 3.3. If $u \in H_{2}^{2}, J(u) \leq C_{0}, k_{g}-\Delta u \leq 0$ and $\int_{M} e^{2 u} d v_{g}=\int_{M} d v_{g}$, there exists a constant $C_{7}$ depending on $C_{0}, k_{g}$ and $\int_{M} d v_{g}$ only such that $\|u\| \leq C_{7}$.

Proof. It is clear from (3.8) and Lemma 3.2 that

$$
\frac{1}{2} e^{-2 C_{2}} \cdot e^{2 \alpha} \cdot \int_{M}(\Delta u)^{2} d v_{g} \leq \frac{1}{2} e^{2 \alpha} \int_{M} e^{-2 u}(\Delta u)^{2} d v_{g} \leq C_{6} .
$$

If we define $C_{7}$ to be $2\left\{2 C_{6} e^{2\left(C_{2}-\alpha\right)}+\left(C_{1}^{2}+C_{2}^{2}\right)\right\}$, combining with Lemma 3.2 , the result follows.

Lemma 3.4. The weak solution of the equation (2.8) exists.

Proof. Let $\alpha_{0}=\inf _{u \in H} J(u)$. Suppose that $\left\{u_{j}\right\}$ is a minimizing sequence in $H_{2}^{2}$ for the functional $J$ with $\int_{M} e^{2 u_{J}} d v_{g}=\int_{M} d v_{g}$. Without loss of generality by applying Lemma 3.1 , we can choose a minimizing sequence $\left\{u_{j}\right\}$ such that $k_{g}-\Delta u_{j} \leq 0$. Then Lemma 3.3 implies that $\left\{u_{j}\right\}$ is bounded in $H_{2}^{2}$. Thus there exists a subsequence of $\left\{u_{j}\right\}$ still denoted by $\left\{u_{j}\right\}$ and a function $u_{0} \in H_{2}^{2}$ such that $u_{j}$ is weakly convergent to $u_{0}$ in $H_{2}^{2}$ and $u_{j}$ is pointwise almost everywhere convergent to $u_{0}$ because $H_{2}^{2}$ is reflexive and the Proposition 3.43 of $[\mathbf{A}]$. Now we have to show that $J\left(u_{0}\right)=\alpha_{0}$ in order to show that $u_{0}$ weakly satisfies the equation (2.8). On the one hand, since the subset $H$ of $H_{2}^{2}$ is weakly closed by Proposition 2.2, $u_{0}$ is in the subset $H$. By definition of $\alpha_{0}$, we can easily see that $\alpha_{0} \leq J\left(u_{0}\right)$. On the other hand, we have

$$
\begin{aligned}
& J\left(u_{n}\right)-J\left(u_{0}\right) \\
& =\int_{M} e^{-2 u_{n}}\left(k_{g}-\Delta u_{n}\right)^{2} d v_{g}-\int_{M} e^{-2 u_{0}}\left(k_{g}-\Delta u_{0}\right)^{2} d v_{g}
\end{aligned}
$$




$$
\begin{aligned}
= & \int_{M}\left(e^{-2 u_{n}}-e^{-2 u_{0}}\right) k_{g}^{2} d v_{g}-2 \int_{M} k_{g}\left(e^{-2 u_{n}} \Delta u_{n}-e^{-2 u_{0}} \Delta u_{0}\right) d v_{g} \\
& +\int_{M}\left[e^{-2 u_{n}}\left(\Delta u_{n}\right)^{2}-e^{-2 u_{0}}\left(\Delta u_{0}\right)^{2}\right] d v_{g} \\
= & \int_{M}\left(e^{-2 u_{n}}-e^{-2 u_{0}}\right) k_{g}^{2} d v_{g}-2 \int_{M} k_{g}\left(e^{-2 u_{n}}-e^{-2 u_{0}}\right) \Delta u_{n} d v_{g} \\
& -2 \int_{M} k_{g} e^{-2 u_{0}} \Delta\left(u_{n}-u_{0}\right) d v_{g}+\int_{M} e^{-2 u_{n}}\left(\Delta\left(u_{n}-u_{0}\right)\right)^{2} d v_{g} \\
& +2 \int_{M} e^{-2 u_{n}} \Delta u_{0} \Delta\left(u_{n}-u_{0}\right) d v_{g}+\int_{M}\left(e^{-2 u_{n}}-e^{-2 u_{0}}\right)\left(\Delta u_{0}\right)^{2} d v_{g} \\
\geq & \int_{M}\left(e^{-2 u_{n}}-e^{-2 u_{0}}\right) k_{g}^{2} d v_{g}-2 \int_{M} k_{g}\left(e^{-2 u_{n}}-e^{-2 u_{0}}\right) \Delta u_{n} d v_{g} \\
- & 2 \int_{M} k_{g} e^{-2 u_{0}} \Delta\left(u_{n}-u_{0}\right) d v_{g}+2 \int_{M}\left(e^{-2 u_{n}}-e^{-2 u_{0}}\right) \Delta u_{0} \Delta\left(u_{n}-u_{0}\right) d v_{g} \\
+ & 2 \int_{M} e^{-2 u_{0}} \Delta u_{0} \Delta\left(u_{n}-u_{0}\right) d v_{g}+\int_{M}\left(e^{-2 u_{n}}-e^{-2 u_{0}}\right)\left(\Delta u_{0}\right)^{2} d v_{g} \\
:= & I+I I+I I I+I V+V+V I .
\end{aligned}
$$

As $n$ goes to $\infty$, I and VI go to zero because $e^{-2 u_{n}}$ goes to $e^{-2 u_{0}}$ pointwise almost everywhere and the Dominated convergence theorem (Theorem 3.32 of $[\mathbf{A}])$ can be applied; III and V go to zero by the definition of the weakly convergence in $H_{2}^{2}$. For II,

$$
\begin{aligned}
& \left|\int_{M} k_{g}\left(e^{-2 u_{n}}-e^{-2 u_{0}}\right) \Delta u_{n} d v_{g}\right| \\
& \leq \max \left|k_{g}\right|\left[\int_{M}\left(\Delta u_{n}\right)^{2} d v_{g}\right]^{1 / 2}\left[\int_{M}\left(e^{-2 u_{n}}-e^{-2 u_{0}}\right)^{2} d v_{g}\right]^{1 / 2} \\
& \rightarrow 0
\end{aligned}
$$

by the Hölder inequality and the Dominated convergence theorem again since $\left\|u_{n}\right\|$ is uniformly bounded. Similarly we can estimate the term IV, we will leave it to reader.

By letting $n \rightarrow \infty$ in (3.10), we have that $\alpha_{0}-J\left(u_{0}\right) \geq 0$, i.e., $J\left(u_{0}\right) \leq \alpha_{0}$. The Lemma is proved.

Remark. In fact, we can show that $J(u)$ is weakly lower semicontinuous on $H_{2}^{2}$. Since we do not need this, we will not give a proof here.

Lemma 3.5. The weak solution of the equation (2.8) is smooth if $k_{g}$ is smooth.

Proof. Suppose $u_{0}$ satisfies (2.8). Set $s=e^{-2 u_{0}}\left(k_{g}-\Delta u_{0}\right)$ and $\lambda^{2}-s^{2}=f$. Then it is clear that equation (2.8) can be written as

$$
\Delta s=e^{2 u_{0}} f .
$$


Since $u_{0} \in H_{2}^{2}, s$ must be square integrable. If we set $v=s-\int_{M} s d v_{g}$, above equation looks like:

$$
\Delta v=e^{2 u_{0}} f .
$$

By applying Theorem 3.67 of $[\mathbf{A}]$ on p. 91 , we can get that $\int_{M}|\nabla v|^{2} d v_{g}$ is finite. Then $v$ is in $L^{p}$ for all $p$ by applying same theorem. Now the above equation tells us that $\Delta v$ is square integrable. Thus $v$, so $s$, is continuous. By using the definition of $s$, we can see that $u_{0}$ is in $C^{2}$. Then $s$ is in $C^{2}$ which will imply that $u_{0}$ is in $C^{4}$. Repeating this argument, we can easily see that $u_{0}$ is smooth. This finishes the proof. In fact, we will see later that $s$ is a constant.

$$
\text { 4. } \chi(M)=0 \text { case. }
$$

In this case, for a given metric $g$ on a Riemmann surface with $\int_{M} k_{g} d v_{g}=0$, we can choose a function $\varphi$ such that $\Delta \varphi=k$ and $\int_{M} e^{2 \varphi} d v_{g}=\int_{M} d v_{g}$ by standard elliptic theory. Now $J(\varphi)=\int_{M} e^{-2 \varphi}\left(k_{g}-\Delta \varphi\right)^{2} d v_{g}=\int_{M} e^{-2 \varphi}\left(k_{g}-\right.$ $\left.k_{g}\right)^{2} d v_{g}=0$. Thus $\varphi$ is a minimizer for $J$. Of course, $\varphi$ is smooth.

\section{The scalar curvatures of the extremal metrics.}

In this section, we will prove the following theroem due to Calabi:

Theorem. For a Riemann surface, the extremal metrics have constant scalar curvatures.

The proof will follow easily from the following

Lemma 5.1. Let $(M, g)$ be a compact Riemann surface. If the Gaussian curvature $k$ of the metric $g$ satisfies

$$
\Delta k+k^{2}=\lambda^{2}
$$

for some constant $\lambda$, then $\nabla k$ is a conformal vector field.

Proof. The $\nabla k$ is a conformal vector field if and only if its components satisfy

$$
k_{i, j}+k_{j, i}=2 f g_{\imath j}
$$

in local coordinates for some function $f$. That is to say that we only need to show that the hessian of the Gaussian curvature is proportional to the metric $g$. The standard Ricci identity shows that

$$
\frac{1}{2} \Delta|\nabla k|^{2}=\sum k_{i j}^{2}+\langle\nabla \Delta k, \nabla k\rangle+R_{i j} k_{i} k_{j} .
$$


But for a Riemann surface, $R_{i j}=k g_{i j}$. Thus

$$
\frac{1}{2} \Delta|\nabla k|^{2}=|\operatorname{Hess}(k)|^{2}+\langle\nabla \Delta k, \nabla k\rangle+k|\nabla k|^{2} .
$$

By using (5.1), we get

$$
\begin{aligned}
\frac{1}{2} \Delta|\nabla k|^{2} & =|\operatorname{Hess}(k)|^{2}-k|\nabla k|^{2} \\
& =|\operatorname{Hess}(k)|^{2}-\frac{(\Delta k)^{2}}{2}+\frac{(\Delta k)^{2}}{2}-k|\nabla k|^{2} .
\end{aligned}
$$

Also by using integration by parts and (5.1) we obtain

$$
\begin{aligned}
\int_{M} k|\nabla k|^{2} d v_{g} & =\int_{M} k \cdot \nabla k \cdot \nabla k d v_{g} \\
& =-\frac{1}{2} \int_{M} k^{2} \Delta k d v_{g} \\
& =\frac{1}{2} \int_{M}\left(\lambda^{2}-k^{2}\right) \Delta k d v_{g} \\
& =\frac{1}{2} \int_{M}(\Delta k)^{2} d v_{g} .
\end{aligned}
$$

Now, we integrate both sides of (5.2) to get

$$
\begin{aligned}
0 & =\int_{M}|\operatorname{Hess} k|^{2} d v_{g}-\int_{M} \frac{(\Delta k)^{2}}{2} d v_{g} \\
& =\int_{M}\left|\operatorname{Hess}(k)-\frac{\Delta k}{2} g\right|^{2} d v_{g} .
\end{aligned}
$$

The last equality holds because of a well known identity. The Lemma can be seen easily from this.

Proof of Theorem. Set $g^{\prime}=e^{2 u_{0}} g$. Then we have $\Delta_{g^{\prime}}=e^{-2 u_{0}} \Delta_{g}$ and $k_{g^{\prime}}=e^{-2 u_{0}}\left(k_{g}-\Delta u_{0}\right)$. Thus the equation (2.8) can be written as

$$
\Delta_{g^{\prime}} k_{g^{\prime}}+k_{g^{\prime}}^{2}=\lambda^{2} \text {. }
$$

Thus Lemma 5.1 can be applied to show that $\nabla k_{g^{\prime}}$ is a conformal vector field with respect to metric $g^{\prime}$. But a conformal vector field $X$ on a manifold $M$ satisfies the identity

$$
\int_{M} X \cdot s_{g} d v_{g}=0
$$

where $s_{g}$ is the scalar curvature of the metric $g$ ([BE],[X]). In our case, $s_{g}=2 k_{g^{\prime}}$. Thus we have

$$
2 \int_{M}\left|\nabla k_{g^{\prime}}\right|^{2} d v_{g^{\prime}}=0
$$


Therefore, $4\left|\nabla k_{g^{\prime}}\right|^{2}=0$. That is, $k_{g^{\prime}}$ is a constant. The theorem is proved.

\section{References}

[A] T. Aubin, Nonlinear Analysis on Manifolds, Monge-Ampère Equations, SpringVerlag, 1982.

[B] M. Berger, On Riemannian structures of prescribed Gaussian curvature for compact 2-manifolds, J. Diff. Geo., 5 (1971), 325-332.

[Be] A. Besse, Einstein Manifolds, Spring-Verlag, 1987.

[BE] J.P. Bourguignon and J.P. Ezin, Scalar curvature functions in a conformal class of metrics and conformal transformations, Trans. AMS, 301 (1987), 723-736.

[BB] D. Burns and P. de Bartolomeis, Stability of vector bundles and extremal metrics, Invent. Math., 92 (1988), 403-407.

[C1] E. Calabi, Extremal Kähler metrics, In Seminar on Differential Geometry (ed. S. T. Yau)(Ann. Math. Study, 102, 259-290), Princeton, Princeton University Press, 1982.

[C2] Extremal Kähler metrics II, In Differential Geometry and Complex Analysis (eds. I. Chavel and H.M. Farkas), Berlin Heidelberg, New York, Springer, 1985, 95114.

[CH] B. Chow, The Ricci flow on the 2-Sphere, J. Diff. Geo., 33 (1991), 325-334.

[H1] R. Hamilton, The Ricci flow on surfaces, In Mathematics and general relativity (ed. J. Isenberg), Contemp. Math., 71, Amer. Math. Soc.

[H2] R. Hamilton, An isoperimetric estimate for the Ricci flow on the two-Sphere, preprint.

[L] M. Levine, A remark on extremal metrics, J. Diff. Geo., 21 (1985), 73-77.

[OPS] B. Osgood, R. Phillips and P. Sarnack, Extremals of determinants of Laplacians, J. Fun. Analysis, 80 (1988), 148-211.

[P] H. Poincaré, Les Functions Fuchsiennes et l'equation $\Delta u=e^{u}$, Oeuvrest II, Gauthier-Villars Paris, (1916), 512-559.

[S] M. Struwe, Variational Methods, Springer-Verlag, 1990.

[X] $\mathrm{X} . \mathrm{Xu}$, On the existence and uniqueness of solutions of Möbius equations, Trans. AMS, 337 (1993), 927-945.

Received November 29, 1993 and revised February 22, 1994.

NATIONAL UNIVERSity OF Singapore

SingAPORE 0511

E-mail address: matxuxw@leonis.nus.sg 
Andreas Seeger, Endpoint inequalities for Bochner-Riesz multipliers in the

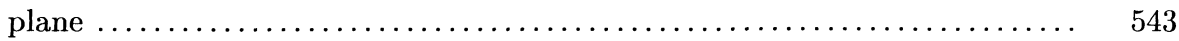

Ted Stanford, Braid commutators and Vassiliev invariants ............ 269

Xiangsheng $\mathbf{X u}$, On the Cauchy problem for a singular parabolic equation 277

Xingwang Xu, On the existence of extremal metrics .............. 555

Rugang Ye, Constant mean curvature foliation: singularity structure and

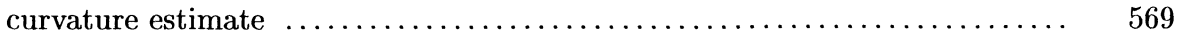




\section{PACIFIC JOURNAL OF MATHEMATICS}

Volume $174 \quad$ No. $2 \quad$ June 1996

Quantum affine algebras and affine Hecke algebras

VyJAYANTHI CHARI and ANDREW PRESSLEY

On the zero sets of bounded holomorphic functions in the bidisc

PHILIPPE CHARPENTIER and JOAQUIM ORTEGA-CERDÀ

Bloch constants in one and several variables

IAN GRAHAM and DROR VAROLIN

Characters of the centralizer algebras of mixed tensor representations of

$G L(r, \mathbb{C})$ and the quantum group $u_{q}(g l(r, \mathbb{C}))$

TOM HALVERSON

Derivations of $C^{*}$-algebras and almost Hermitian representations on

$\Pi_{k}$-spaces

EDWARD Kissin, ALEKSEI I. LOGINOV and Victor S. SHULman

Twisted Alexander polynomial and Reidemeister torsion

TERUAKI KITANO

Explicit solutions for the corona problem with Lipschitz data in the polydisc

STEVEN KRANTZ and SONG-YING Li

Prepolar deformations and a new Lê-Iomdine formula

DAVID MASSEY

$K K$-groups of twisted crossed products by groups acting on trees

Kevin Paul McClanahan

Coherent states, holomorphic extensions, and highest weight representations

KARL-HERMANN NEEB

Endpoint inequalities for Bochner-Riesz multipliers in the plane

ANDREAS SEEGER

On the existence of extremal metrics

XINGWANG XU

Constant mean curvature foliation: singularity structure and curvature estimate 569 RUGANG YE 